\title{
Surface Analysis of Zeolites: An XPS, Variable Kinetic Energy XPS, and Low Energy Ion Scattering Study
}

Simon R. Bare ${ }^{1 \#}$, Axel Knop-Gericke ${ }^{2}$, Detre Teschner ${ }^{2}$, Michael Hävacker ${ }^{2}$, Raoul Blume $^{2}$, Tulio Rocha ${ }^{3}$, Robert Schlögl ${ }^{2}$, Ally S.Y. Chan ${ }^{1}$, N. Blackwell ${ }^{1}$, M.E. Charochak ${ }^{1}$, Rik ter Veen ${ }^{4}$, and Hidde H. Brongersma ${ }^{5}$

${ }^{1}$ UOP LLC, a Honeywell Company, Des Plaines, IL 60017, USA

${ }^{2}$ Fritz-Haber-Institut der Max-Planck-Gesellschaft, Faradayweg 4-6, 14195 Berlin, Germany

${ }^{3}$ Laboratório Nacional de Luz Síncrotron, Campinas, Brazil

Formatiert: Englisch (USA)

${ }^{4}$ Tascon GmbH, Mendelstrasse 17, 48149 Münster, Germany

${ }^{5}$ Eindhoven University of Technology, P.O. Box 513, 5600 MB Eindhoven, The Netherlands

"Corresponding author, e-mail: simon.bare@honeywell.com

\begin{abstract}
The surface Si/Al ratio in a series of zeolite Y samples has been obtained using laboratory XPS, synchrotron (variable kinetic energy) XPS, and low energy ion scattering (LEIS) spectroscopy. The nondestructive depth profile obtained using variable kinetic energy XPS is compared to that from the destructive argon ion bombardment depth profile from the lab XPS instrument. All of the data indicate that the near surface region of both the ammonium form and steamed $Y$ zeolites is strongly enriched in aluminum. It is shown that when the inelastic mean free path of the photoelectrons is taken into account that the laboratory XPS of aluminosilicates zeolites does not provide a true measurement of the surface stoichiometry while using variable kinetic energy XPS a more surface sensitive measurement can be made. A comprehensive Si/Al concentration profile as a function of depth is developed by combining the data from the three surface characterization techniques. The LEIS spectroscopy reveals that the topmost atomic layer is even further enriched in Al.
\end{abstract}

\section{Introduction}

The acidity/hydrophobicity, and therefore the activity, of an aluminosilicate zeolite are determined by the aluminum content and the location of the $\mathrm{Al}$ in the zeolite crystal. Knowledge of the location of the Al is therefore fundamental to understanding the zeolite activity. Moreover, the external surface of zeolite particles plays an important role in much catalytic chemistry as large reactant molecules react 
predominantly at the pore mouth of the zeolite (e.g. in fluid catalytic cracking), as they cannot enter into the smaller channels. Indeed pore-mouth catalysis has been proposed to explain the observed selectivity in selective isomerization, transalkylation, and hydrocarbon cracking [1-3]. As such it is no surprise that the characterization of the surface composition of zeolites has been the subject of much study, particularly using x-ray photoelectron spectroscopy (XPS) [4-7] and also using low energy ion scattering spectroscopy (LEIS) [8].

However, in conventional laboratory-based XPS, with a fixed excitation source (typically either $\mathrm{Mg} \mathrm{K \alpha}$ $(1253.6 \mathrm{eV})$ or Al K $\alpha(1486.6 \mathrm{eV}))$, the high inelastic mean free path (IMFP) of photoelectrons with low binding energy (e.g. Al $2 p$ with a binding energy of $\sim 74 \mathrm{eV}$ ) results in a relative lack of true surface sensitivity to the outer few atomic layers. Additionally, the laboratory XPS measurement results in a single value of the elemental concentration for each sample, with no information provided on the presence of any depth concentration profile. However, a depth concentration profile using a laboratory XPS can be obtained using argon ion sputter depth profiling to sputter away the surface layers on a pseudo layer-by-layer manner. Thus, by repeated cycles of sputtering and measurement a sputter depth profile is obtained. For smooth surfaces (e.g. thin films) a sharp concentration profile can be obtained [9], but for powdered materials the situation is distinctly more complex as the true "depth" is not accurately known for such a macroscopically rough surface. Ar ion sputtering is also complicated by the preferential sputtering of one element over another which makes quantification more difficult [9]. A second method for determining if there is preferential concentration of one element closer to the surface using XPS is to measure the signal as a function of angle, taking advantage of the enhancement of the signal from the surface at grazing exit angles $[9,10]$. There have been reports of such studies providing concentration profiles from irregular surfaces such as powdered materials [11, 12].

A non-destructive depth profile, with increased surface sensitivity, can be obtained by varying the kinetic energy of the photoelectrons in XPS, using the so-called variable kinetic energy XPS. The kinetic energy is varied by varying the photon energy of the $x$-ray beam by using a monochromatic $x$-ray beamline at a synchrotron radiation source. This method has been applied to the study of e.g. a 100nm Ta layer with native oxide, a Sn-doped $\ln _{2} \mathrm{O}_{3}$ single crystal and $\mathrm{Sn}$-doped $\operatorname{In}_{2} \mathrm{O}_{3}$ powder [13], and to the study of catalyst surfaces under reaction conditions [14]. The development and rapid expansion of ambient pressure XPS at synchrotron sources around the globe has ensured that there are now many beamlines that are capable of providing monochromatic variable kinetic energy $x$-rays [15]. There are only a few studies that have taken advantage of this capability for the surface analysis of zeolites, and we know of no studies that combine and compare the data from multiple XPS methods, together with that from low energy ion scattering (LEIS). Shimada et al. [16-18] used synchrotron radiation XPS to determine the surface composition of $\mathrm{NaY}$ and $\mathrm{HY}$ zeolites. They concluded that there was a thin Al-rich overlayer on the external surface of $\mathrm{NaY}$, and a gradual increase in the $\mathrm{Si} / \mathrm{Al}$ ratio from the external surface for HY. Shimada et al. [17] compared the XPS depth profiling Si/Al ratio of NaY and HY using destructive (Ar+ sputtering) with non-destructive (synchrotron XPS). They concluded that both methods gave consistent $\mathrm{Si} / \mathrm{Al}$ profiles.

In this work XPS, angle-resolved XPS, sputter depth profiling XPS, variable kinetic energy XPS, and low energy ion scattering (LEIS) are used to determine the surface concentration and depth profile of

Page $\mathbf{2}$ of $\mathbf{1 7}$ 
aluminum in a series of zeolite $Y$ samples with the aim of comparing and contrasting the information content obtained from each method. Zeolite $Y$ is a critically important zeolite used in petroleum refining. It is the primary zeolitic constituent, and therefore the component that provides the necessary Brønsted acidity, of fluid catalytic cracking (FCC) catalysts, a \$1 billion per year catalyst market, and provides the greatest gasoline yield at the highest octane combined with the best stability [19]. The active material is typically prepared in the sodium form, $\mathrm{NaY}$. The sodium is then ion exchanged out of the structure using ammonium hydroxide. It is then steam calcined, followed by a second ion exchange to remove the final traces of sodium, followed by a second steam calcination step. This procedure is known to produce active and stable form of zeolite $Y[20]$.

\section{Experimental}

\section{Samples}

The samples used in this study were $\mathrm{NaY}, \mathrm{NH}_{4}, \mathrm{H}$-deAl-Y and US-Y powders, all with a bulk Si/Al ratio determined by ICP of 2.6, were synthesized at UOP. The $\mathrm{NH}_{4}, \mathrm{H}$-deAl-Y was prepared by three times ion exchange of the parent sodium form, $\mathrm{NaY}$, followed by calcination and steaming at $600^{\circ} \mathrm{C}$ for 2 hours followed by an additional three times ion exchange. The US- $Y$ was prepared from the $\mathrm{NH}_{4}, \mathrm{H}$-deAl-Y sample by further steaming at $735^{\circ} \mathrm{C}$ for 2 hours. All of the powders were highly crystalline by XRD. The typical particle size is $1.5 \mu \mathrm{m}$ diameter determined by Sedigraph and $<1 \mu \mathrm{m}$ by SEM. Figure 1 is a schematic showing the evolution of the different samples.

\section{Synthesis $\Rightarrow$ Ion exchange $\Rightarrow$ Steam calcine $\Rightarrow$ Ion exchange}

Na-Y $\quad \mathrm{NH}_{4}, \mathrm{Na}-\mathrm{Y} \quad \mathrm{H}, \mathrm{Na}-\mathrm{deAl}-\mathrm{Y}$

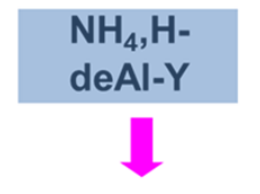

Steam calcine

US-Y

Figure 1. Schematic illustrating the processing of the parent zeolite leading to the other zeolite samples studied.

Figure 2 shows a representation of the structure of the $\mathrm{Y}$ zeolite, which is also known as Faujasite, with the designation FAU. The unit cell is cubic with length $24.7 \AA$ 

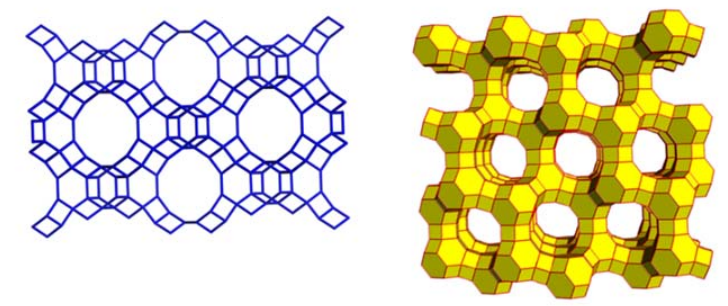

Figure 2. Structure of the unit cell of Faujasite. The image (left) shows a projection of $a=2, b=1, c=1$ along the [011] direction. The unit cell size is $24.7 \AA$

\section{X-ray Photoelectron Spectroscopy}

The laboratory XPS data were obtained using a PHI Quantum 2000 Scanning ESCA Microprobe ${ }^{\mathrm{TM}}$

(Physical Electronics, Inc.) with a micro-focused, monochromatic Al K $\alpha$ X-ray source at $1486.6 \mathrm{eV}$. A dual neutralization capability using low energy electrons and positive ions to provide charge compensation during spectral acquisition is standard in this instrument. All XPS spectra are recorded at room temperature without sample pretreatment. The XPS data were collected from powders adhered to double-sided tape for the basic measurements, (the thickness of the layer of powder was such that the peaks from the tape were not observed) and from samples hand-pressed into $7 \mathrm{~mm}$ diameter pellets for the angle resolved and sputter depth profile measurements. Sputter depth profiles are generated by alternating cycles of spectral acquisition of the sample surface, followed by $2 \mathrm{kV} \mathrm{Ar}^{+}$bombardment of the sample surface for 15 to 30s in each cycle. The sputter depth rate is calibrated using an atomically smooth silica film on a silicon wafer of known thickness. This calibration does not take any preferential sputtering into account.

The synchrotron (ambient pressure) XPS experiments were performed in the NAP-XPS setup at the ISISS beamline of the FHI located at the BESSY II synchrotron radiation facility in Berlin, Germany. The setup consists of a reaction cell attached to a set of differentially pumped electrostatic lenses and a separately pumped analyzer (Phoibos 150 Plus, SPECS GmbH), as described elsewhere[21]. XPS spectra were collected at a temperature of $225^{\circ} \mathrm{C}$ and in the presence of 0.5 mbar $\mathrm{H}_{2} \mathrm{O}$. The spectra were collected at elevated temperature and in a partial pressure of $\mathrm{H}_{2} \mathrm{O}$ to better simulate the conditions under which the zeolites are used in catalysis, and this also had the added benefit of reducing sample charging during XPS data collection. Integrated intensity of Si2p and Al2p corrected for cross-section [22] as a function of photon energy, flux as function of energy, and beam current [23]. The zeolite samples were dispersed on a gold foil and mounted in the spectrometer. The base pressure in the BESSY system is typically 
higher than that in the laboratory XPS and often results in an increase in the C 1s signal from adventitious carbon compared to the equivalent sample measured in the laboratory system.

\section{Low Energy Ion Scattering}

The low energy ion scattering data were measured at ION-TOF GmbH with the Qtac ${ }^{100}$ High Sensitivity LEIS (HS-LEIS) instrument [24-26]. To remove any environmental contamination, the samples were first treated in the instrument with atomic oxygen of thermal energy (thus no sputtering) for a period of 10 $\min$. A static analysis is ensured by rastering the $5 \mathrm{nA} \mathrm{He}{ }^{+}$ion beam over a $2 \times 2 \mathrm{~mm}^{2}$ sample area. The typical measurement time was 3 minutes, with an applied ion fluence of $1.4 \times 10^{14} \mathrm{~cm}^{-2}$.

An important goal of the present study is to distinguish and quantify the atomic concentrations of $\mathrm{Al}$ and Si. Since these elements are adjacent in the periodic table (primary masses 27 and 28 amu, respectively), this is difficult. Using conventional LEIS equipment the separation of $\mathrm{Al}$ and $\mathrm{Si}$ is impossible $[27,28]$. The $\operatorname{Qtac}^{100}$, which is a dedicated LEIS instrument, combines a well-defined scattering angle $\left(145^{\circ}\right)$ with a large solid angle of acceptance, parallel energy detection and a wide energy range $(0.5-8 \mathrm{keV})[25,26]$. Since at low primary energies $E_{o}$ the width of a LEIS peak is determined by inelastic processes, it scales with $\mathrm{VE}_{0}$, while the energy separation of the $\mathrm{Al}$ and $\mathrm{Si}$ peaks is proportional to $\mathrm{E}_{\mathrm{o}}$. Thus the peaks are relatively sharper for higher primary energies. Here $6 \mathrm{keV} \mathrm{He}^{+}$is used where the scattered ion energy is $\sim 3400 \mathrm{eV}$, which still ensures a selective analysis of the outer atomic layer. For the quantitative evaluation the $\mathrm{Al}$ and $\mathrm{Si}$ the signals are compared with those of pure standards $\left(\mathrm{Al}_{2} \mathrm{O}_{3}, \mathrm{SiO}_{2}\right)$. Since it is difficult to obtain $\mathrm{Na}_{2} \mathrm{O}$ as reference for sodium, the sensitivity for $\mathrm{Na}$ was derived from a linear correlation plot. For a two component system this is a straight line [25], and a plane in the present case of three components. This calibration gives the surfaces areas that are covered by $\mathrm{Al}_{2} \mathrm{O}_{3}, \mathrm{SiO}_{2}$ and $\mathrm{Na}_{2} \mathrm{O}$. Taking into account the error in the peak fitting procedure for $\mathrm{Al}$ and $\mathrm{Si}$, the error of these coverages is estimated to be around $5 \%$. These values are converted to the atomic surface composition using the surface areas of $\mathrm{AlO}_{1.5}, \mathrm{SiO}_{2}$ and $\mathrm{NaO}_{0.5}$.

Even at $6 \mathrm{keV}$ the energy difference between the $\mathrm{Al}$ and Si peaks is only $69 \mathrm{eV}$, so there is still a strong overlap. A potential danger for this analysis is the presence of surface charging, which will shift the energies of the peaks. Especially inhomogeneous charging must be avoided. This can be achieved by heating the sample (above $570 \mathrm{~K},[29]$ ), or with an electron shower [27, 30]. Here it is done with a special low-energy electron shower and the charge compensation is verified by checking the energy distribution of the sputtered ions near $0 \mathrm{eV}$ in the LEIS spectrum[30]. An example of the raw data obtained from the NaY sample is shown in the Supplemental Information (Fig. S1).

Depth profiles (Fig. 7) are obtained by alternating the static $\mathrm{He}^{+}$LEIS analysis with $\mathrm{Ne}^{+}$sputtering $(5 \mathrm{keV}$, $25 \mathrm{nA}$ ). The depth scale is based on the assumption that two atoms are sputtered per incident Ne-ion. In order to keep the analytical conditions constant, the sample were re-oxidized with atomic oxygen before each analysis.

Low energy ion scattering (LEIS) is the most surface sensitive analytical technique as the signal only originates from the topmost atomic layer [26]. This is in contrast even to the variable kinetic energy XPS experiment where the measured signal is still an integral over the IMFP, as described above. Thus, the 
$\mathrm{Si} / \mathrm{Al}$ ratio of the first atomic layer of the zeolites LEIS data were collected for the NaY and $\mathrm{H}$-deAl-Y samples, and the data are presented in Figure 7. In order to obtain a depth profile sputtering has to be used, as was done for the lab XPS.

\section{Results \& Discussion}

The surface Si/Al ratio of the three zeolite powders are presented in Table I, as measured by the laboratory XPS instrument. The value measured by XPS is different from the bulk value of these zeolites, 2.6, determined using inductively coupled plasma spectroscopy. The surface of the NaY starting material is slightly depleted in Al compared to the bulk, whereas the ammonium-exchanged and calcined sample, and the stabilized US-Y sample are both enriched in Al compared to the bulk value.

Table I. Surface Si/Al ratio of the zeolite powders measured using Al Ka radiation

\begin{tabular}{|l|c|}
\hline Sample & Si/Al rato \\
\hline Na-Y & $\mathbf{3 . 0}$ \\
\hline NH$_{4}, \mathbf{H}-$ deAl-Y & $\mathbf{2 . 0}$ \\
\hline US-Y & $\mathbf{1 . 3}$ \\
\hline
\end{tabular}

These values represent an average over the sampling depth of the laboratory XPS measurement using AI $\mathrm{K} \alpha$ radiation and there is no information on any gradient in the $\mathrm{Si} / \mathrm{Al}$ ratio in the near surface region. One method to determine depth profile information using XPS is to use argon ion bombardment, i.e. $\mathrm{Ar}^{+}$ sputtering. XPS data are acquired on the starting material, and after fixed amounts of sputtering time to remove layers of the material. The results of such an experiment for the three zeolite powders under study are presented in Figure 3.

The data show that the NaY sample appears to be depleted in Al at surface, the $\mathrm{NH}$, H-deAl-Y sample is enriched in $\mathrm{Al}$ at surface, and the US-Y sample is strongly enriched in Al at surface, compared to the bulk value of 2.6. The Si/Al profile for the $\mathrm{NH} 4 \mathrm{H}$-deAl-Y rapidly reaches its asymptote at close to the bulk value, whereas those for the NaY and US-Y more gradually approach the asymptote. 


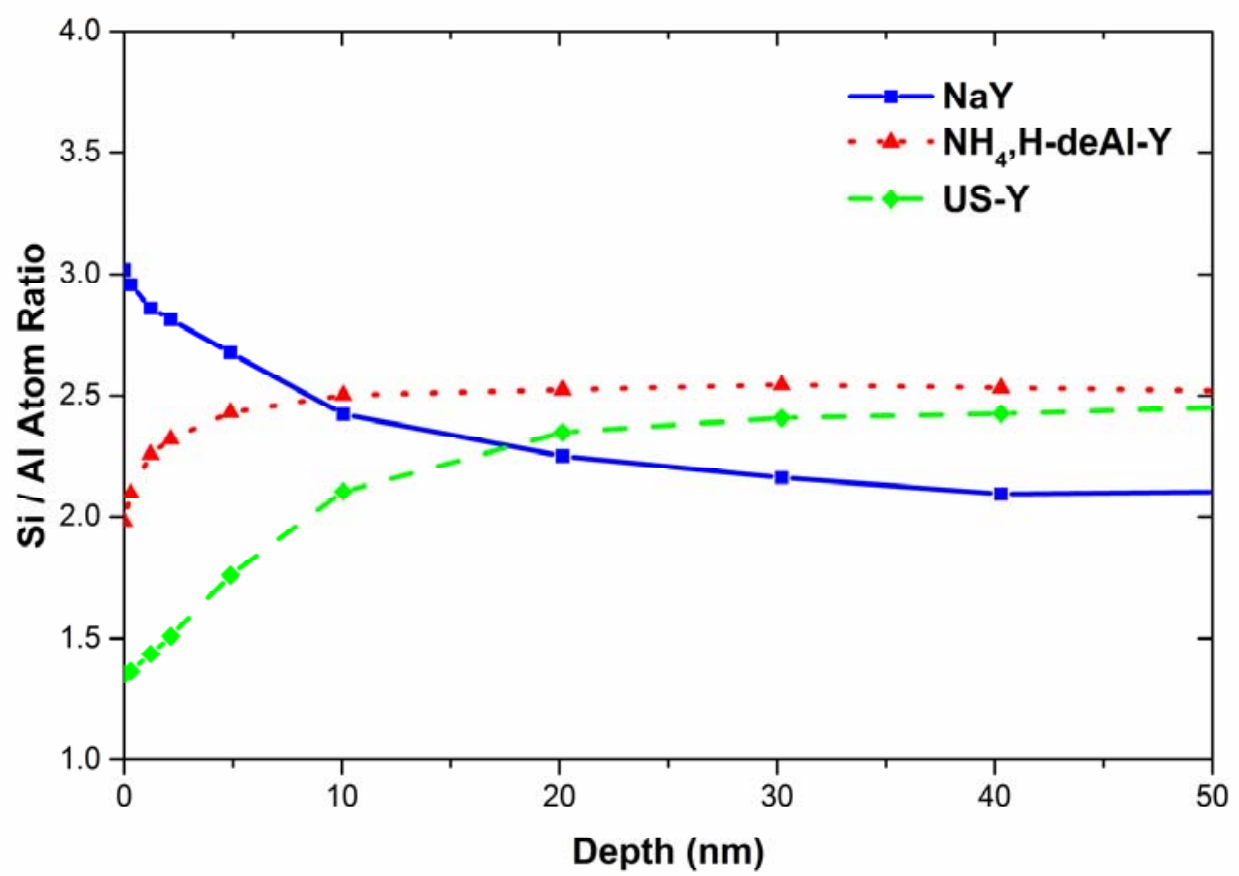

Figure 3. Laboratory XPS Ar+ sputter depth profile of the three zeolite powders. NaY blue squares, $\mathrm{NH} 4, \mathrm{H}-\mathrm{deAl}-\mathrm{Y}$ red triangles, US-Y green diamonds. The depth scale is approximate and was calibrated using a thin silica film on silicon.

It is well known that argon ion sputter depth profiling has some deficiencies and the data must be interpreted with care. These deficiencies include preferential sputtering, charging, and issues with quantitatively knowing the depth, particularly from surfaces that are not macroscopically smooth like the zeolite powders used here. There have been reports of preferential sputtering for zeolites [18]. In this study the fact that the $\mathrm{Si} / \mathrm{Al}$ ratio approaches the known bulk value suggests that preferential sputtering is not a significant concern for the $\mathrm{NH}_{4}, \mathrm{H}$-deAl-Y and US-Y samples, but the presence of the sodium in the $\mathrm{NaY}$ material may have an effect on the relative removal of the $\mathrm{Si}$ or $\mathrm{Al}$ in this sample as the Si/Al ratio for this material drops below that of the bulk value. There is also some uncertainty in the depth axis as the depth axis of the sputter depth profile is determined from a calibration using a dense atomically smooth film of $\mathrm{SiO}_{2}$ on a $\mathrm{Si}$ wafer. The zeolite powder samples are rough, and the sample is porous. Both of these will affect the sputter depth.

A non-destructive method that can be used to determine depth information using XPS is angle resolved XPS. It has been shown that on flat substrates the surface sensitivity can be increased by an order of 
magnitude at grazing exit angles, and that this method can also be applicable to irregular surfaces [11, 12]. Angle-resolved XPS data were collected from the three zeolite samples using the lab XPS instrument, and the results are presented in Figure 4.

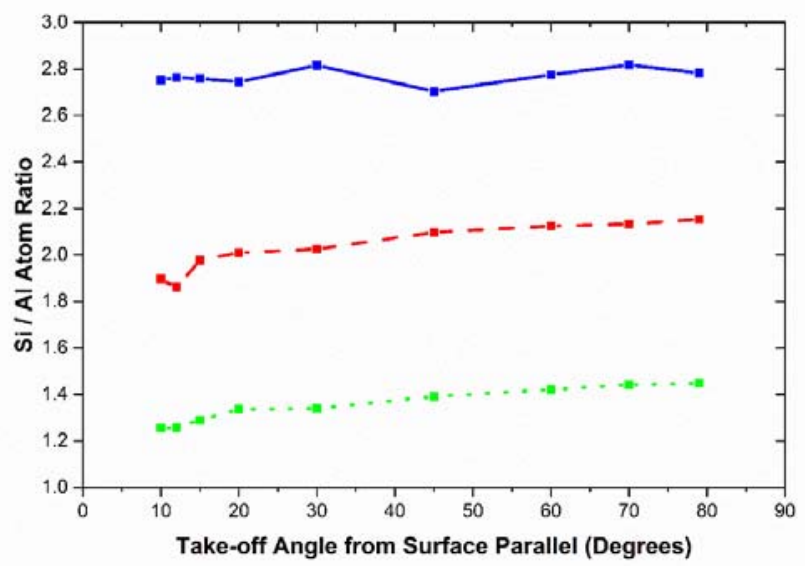

Figure 4. Si/Al ratio as a function of the take-off angle. NaY blue solid line, NH4, H-deAl-Y red dash line, US-Y green dotted line.

In the laboratory instrument used in this study the angle between the x-ray and the analyzer is fixed at $45^{\circ}$. The data shown in Fig. 4 were obtained by rotating the sample with respect to the analyzer, and thus this also changes the angle of the incident $x$-ray beam. Nevertheless, the data shown in Fig. 4 are consistent with the Si/Al ratio of the $\mathrm{NH}_{4}, \mathrm{H}-\mathrm{deAl}-\mathrm{Y}$ and US- $\mathrm{Y}$ decreasing towards grazing exit (low takeoff angle, more surface sensitive), whilst that from the NaY appears to be insensitive to the angle. However, it is noted that these changes are small, and a changes of less than $10 \%$ are within the measurement error. Qualitatively these data are in agreement with the sputter depth profile data (Fig. 3) for the $\mathrm{NH}_{4}, \mathrm{H}$-deAl-Y and US-Y zeolites. The laboratory XPS-derived surface Si/Al ratio is different from the bulk value for all of the samples.

The data presented thus far were collected with a laboratory XPS spectrometer with fixed incident monochromatic x-ray energy $(1486.6 \mathrm{eV})$. In XPS the probability that a photoelectron will travel a distance, $d$, without suffering inelastic scattering is $\exp (-d / \lambda$,) where $d$ is depth and $\lambda$, the inelastic mean free path. This implies that the majority of signal originates from a depth of $1 \lambda$, and $95 \%$ from $3 \lambda$. This is the so-called "information depth" of XPS. It is instructional to calculate the inelastic mean free path (IMFP) for a material similar to the zeolitic samples studied here. The resulting IMFP for silica is plotted in Figure 5 as a function of kinetic energy of the photoelectron. 


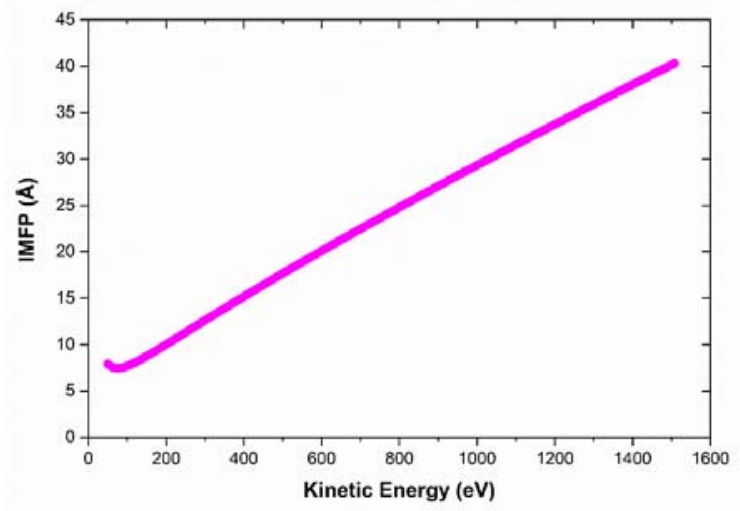

Figure 5. Inelastic mean free path for $\mathrm{SiO} 2$ calculated using Tanuma, Powell \& Penn, TPP=2M [31].

Using $1486.6 \mathrm{eV}(\mathrm{AIK} \alpha)$ as the incident $\mathrm{x}$-ray energy the resulting Si2p photoelectron has a kinetic energy

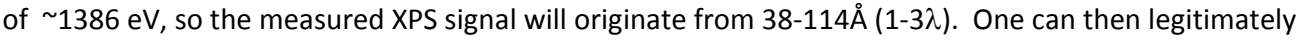
ask if such a measurement can be called "surface" analysis. For a material only comprising low atomic number elements like these aluminosilicates zeolites the laboratory XPS is not truly "surface" sensitive, especially when the photoelectron being measured has a low binding energy, as is the case of for the $\mathrm{Si} 2 \mathrm{p}$ and AI2p photoelectron lines that are commonly used to characterize the surface composition of zeolites.

Using a photon source with variable photon energy, like a beamline at a synchrotron light source, the kinetic energy of the resulting photoelectron can be tuned so that the information depth then becomes a variable in the experiment. This would provide the advantages that the measurement could be both more surface sensitive by using a lower photon energy than the lab source, and by varying the photon energy, the resulting depth profile would be non-destructive. The latter would be in sharp contrast to the sputter depth profile measurement, and may alleviate some of the unknowns e.g. regarding preferential sputtering.

Figure 6 shows comparative XPS data from the lab instrument (photon energy of $1486.6 \mathrm{eV}$ ) and the synchrotron (using a photon energy $1210 \mathrm{eV}$ ) from the sample $\mathrm{NaY}$. As can be seen the data quality obtained at the beamline is comparable to that obtained using the laboratory instrument - but the data acquired at the synchrotron were additionally acquired in a partial pressure of water vapor of $0.5 \mathrm{mbar}$ and at a temperature of $225^{\circ} \mathrm{C}$ - so a step towards operando conditions compared to the UHV and room temperature environment of the laboratory XPS data. 

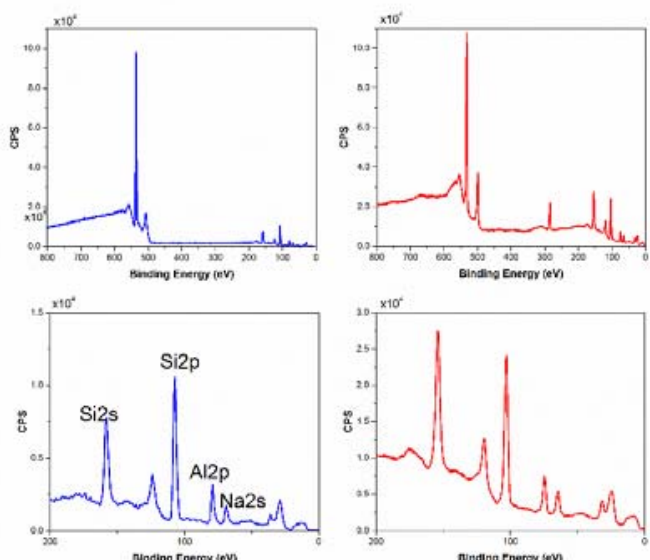

Figure 6. Comparison of Lab XPS (right) with Synchrotron XPS (left) for the NaY sample. Survey scans (top), and Si2p and AI2p regions (bottom).

Table II compares the surface Si/Al ratio from the three zeolite samples obtained from the synchrotron XPS and the laboratory XPS using a comparable photon energy (similar information depth). The surface $\mathrm{Si} / \mathrm{Al}$ ratio of the three zeolite samples are reasonable agreement - but now the photon energy can be varied with the ambient pressure instrument at the synchrotron in order to vary the probe the depth non-destructively.

Table II. Comparison of Si/Al ratio of Lab XPS (1486.6 eV) and synchrotron XPS (1210 eV)

\begin{tabular}{|l|c|c|}
\hline \multicolumn{2}{|c|}{ Si/Al ratio } \\
\hline Na-Y & $\begin{array}{c}\text { Lab XPS } \\
(1486.6 \mathrm{eV})\end{array}$ & $\begin{array}{c}\text { Synchrotron XPS } \\
(1210 \mathrm{eV})\end{array}$ \\
\hline NH $_{4}, \mathrm{H}-$ deAl-Y & 3.0 & 2.77 \\
\hline US-Y & 2.0 & 2.3 \\
\hline
\end{tabular}

Figure 7 shows a plot of the Si/Al ratio as a function of IMFP (obtained by varying the photon energy) from the three zeolite powders. This is thus a true non-destructive depth profile over the top $40 \AA$ of the zeolite surface, and moreover the data were obtained under conditions that are more appropriate for some catalytic reactions (in this case $225^{\circ} \mathrm{C}$ and $0.5 \mathrm{mbar}_{2} \mathrm{O}$ ). 


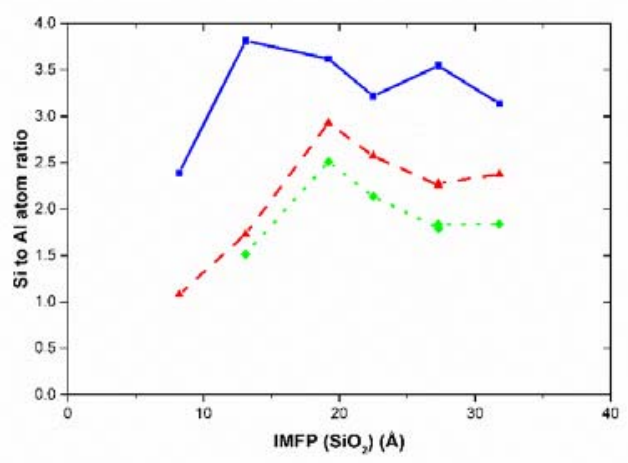

Figure 7. Variable kinetic energy XPS derived Si/Al ratio versus IMFP for the three zeolite samples. NaY blue squares, $\mathrm{NH}{ }_{4}, \mathrm{H}$-deAl$Y$ red triangles, US-Y green diamonds measured at $225^{\circ} \mathrm{C}$ and in the presence of $0.5 \mathrm{mbar} \mathrm{H}_{2} \mathrm{O}$.

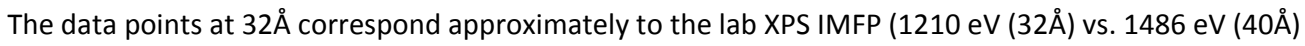
photon energy), and all data at lower IMFP are new information that was not possible to obtain with the laboratory instrument, and only possible from the ability to vary the photon energy of the $x$-ray beam to lower energy. First, the data are consistent with the lab XPS in that the SI/Al ratio of the $\mathrm{NaY}$ is greater than that of the $\mathrm{NH}_{4}, \mathrm{H}$-deAl-Y, which is greater than the US-Y (see Fig. 2). The synchrotron XPS data show that the very outer surface $(<10 \AA)$ of both the $\mathrm{NH}_{4}, \mathrm{H}$-deAl-Y and USY is enriched in aluminum relative to immediate sub-surface. The $\mathrm{Si} / \mathrm{Al}$ ratio of these samples increases at around $20 \AA$ and then drops again before leveling out. It is tempting to link the maximum observed in these profiles with the unit cell size of the $Y$ zeolite, which is approx. $25 \AA$. . However, it is unclear at this stage why there would be such a maximum. The $\mathrm{Si} / \mathrm{Al}$ profile of the $\mathrm{NaY}$ is flatter than that of the other samples, but also shows an enrichment of $\mathrm{Al}$ at the very surface.

Low energy ion scattering (LEIS) is the most surface sensitive analytical technique as the signal only originates from the topmost atomic layer [26]. This is in contrast even to the variable kinetic energy XPS experiment where the measured signal is still an integral over the IMFP, as described above. Thus, the $\mathrm{Si} / \mathrm{Al}$ ratio of the first atomic layer of the zeolites LEIS data were collected for the $\mathrm{NaY}$ and $\mathrm{NH}_{4}, \mathrm{H}-\mathrm{deAl}-\mathrm{Y}$ samples, and the data are presented in Figure 8. The raw data used to generate the profile for $\mathrm{NaY}$ is shown in the Supplemental Information. The raw LEIS spectra In order to obtain a depth profile sputtering has to be used, as was done for the lab XPS. In the LEIS instrument the depth axis obtained by dynamic neon sputtering of the sample. 


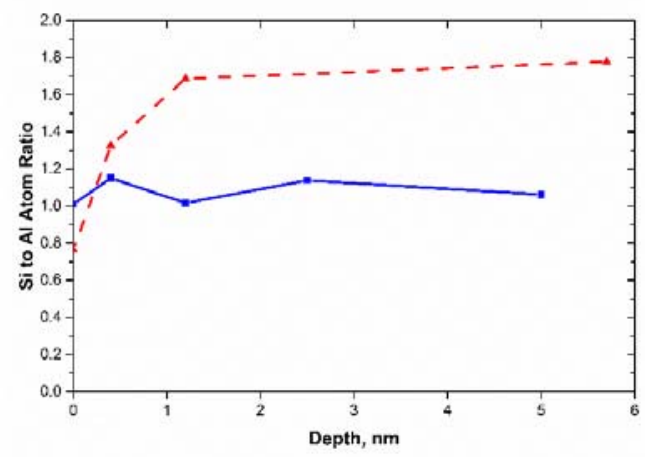

Figure 8. Depth profile of the Si/Al atomic ratio of $\mathrm{NaY}$ (blue squares) and $\mathrm{NH}_{4}, \mathrm{H}$-deAl-Y (red triangles) as determined by $6 \mathrm{keV}$ ${ }^{4} \mathrm{He}^{+}$ion scattering.

The LEIS data show a dramatic surface enrichment of $\mathrm{Al}$ in the topmost layer in both the $\mathrm{NaY}$ and $\mathrm{NH}_{4}, \mathrm{H}-$ deAl-Y samples, with a Si/Al ratio of less than unity for the $\mathrm{NH}_{4}, \mathrm{H}$-deAl-Y sample and close to unity for the $\mathrm{NaY}$ sample. When the sputtering data are taken into account the Si/Al ratio for the $\mathrm{NH}_{4}, \mathrm{H}-\mathrm{deAl}-\mathrm{Y}$ sample increases quickly within $1.5 \mathrm{~nm}$ before leveling off, whereas the profile for the NaY sample is relatively flat. Since the LEIS depth profiling was done with rather high energy ( $5 \mathrm{keV}) \mathrm{Ne}^{+}$ions, the subsurface results will be affected by some ion beam mixing. In reality the increase of the Si/Al for $\mathrm{NH}_{4}, \mathrm{H}-$ deAl-Y will thus be even steeper than seen in Fig. 8.

When sputter depth profiling alkali containing glasses, the irradiation will cause an increased vacancy concentration and enhanced alkali diffusion [32]. The alkali will surface segregate, where they are sputtered away. This will lead to an alkali depleted layer over the penetration depth of the incident ions [32]. All techniques that use sputtering for depth profiling will be affected. The LEIS determination of the Na concentration in the outer surface ( 14 at.\% vs. the bulk ICP value of $11.3 \mathrm{wt} \%$ ) of the NaY zeolite is determined before sputtering and thus not influenced.

If the data from the LEIS, synchrotron XPS and lab XPS are compared then some general trends can be observed. All three methods are consistent in that there is an enrichment of $\mathrm{Al}$ in the near surface region for the $\mathrm{NH}_{4}, \mathrm{H}$-deAl-Y and USY samples, and that with increasing depth into the sample the ratio increases and eventually approaches that of the bulk value. Similarly the methods show that the Si/Al 6 $\mathrm{keV} 4 \mathrm{He}+$ ion scattering depth profile for the $\mathrm{NaY}$ is flatter than the other two samples.

In an attempt to reconcile the data from the different surface analytical methods used to determine the surface composition and any depth distribution of the Si/Al ratio of the zeolite $\mathrm{Y}$ samples, the data from the LEIS, synchrotron XPS and lab XPS for all three samples are plotted and summarized in Figure 9 . The data in the upper panel of Fig. 8 are plotted on the same depth axis, using the original depth data from Figs 3, 7 and 8. It is difficult to see the linkage between the different surface analytical methods and any trends in this plot. However, if the information depth (IMFP) of the XPS measurement is taken into account, and the depth of the lab XPS shifted by $4 \mathrm{~nm}$ and re-plotted (Figure 8 lower panel), then the 
picture becomes clearer. The data in Fig. 8 lower panel now appears to be a continuous profile in the $\mathrm{Si} / \mathrm{Al}$ ratio from the surface into the bulk of the sample for all three zeolite samples. Indeed the agreement between the last point from the synchrotron XPS and the first point from the lab XPS, and the continuity of the profile is quite remarkable. If the Si/AI ratio of the synchrotron XPS data in Fig. 9 (lower panel) are extrapolated to the very top surface then for the two samples where there is LEIS data $\left(\mathrm{NH}_{4}, \mathrm{H}\right.$-deAl-Y and $\left.\mathrm{NaY}\right)$ then there is also agreement in the expected value between the extrapolated XPS values and the initial top surface LEIS values: there is a continued surface concentration of AI.

However, there does appear to be a discrepancy between the absolute values of the Si/Al ratio between the sputter profile LEIS data and the synchrotron XPS data for the NaY sample. The cause of this discrepancy is not known at this time, and will be the focus of future work.
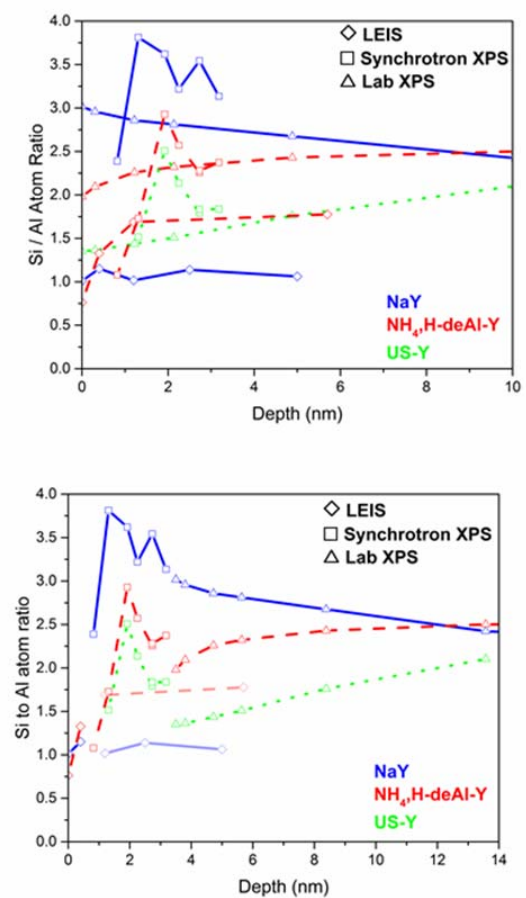

Figure 9. Data from the LEIS (open diamonds), synchrotron XPS (open squares), and lab XPS (open triangles) from the NaY (blue), $\mathrm{NH}_{4}, \mathrm{H}$-deAl-Y (red), and USY (green) zeolite samples. The data from the lab XPS sputter depth profile have been offset $4 \mathrm{~nm}$ and the sputter points from the LEIS shaded out in the lower panel.

\section{Summary}

Page 13 of 17 
Knowledge of the surface concentration of the Brønsted acid sites in a zeolite is fundamental to understanding the reactivity of this important class of solid acid catalyst, particularly for reactions that may occur at the pore mouth. In this work, surface characterization data from a series of $Y$ zeolites have been collected using laboratory XPS, synchrotron XPS (variable kinetic energy), and low energy ion scattering spectroscopy and the information obtained from these methods compared and contrasted. It is shown that the laboratory XPS measurement does not provide a true analysis of the outermost surface of zeolites, but that this information can be obtained using variable kinetic energy XPS combined with LEIS. In the future we plan to extend this work to other families of zeolites and also, via the use of new beamlines that are being developed that allow XPS data to be collected using photon energy up to $\sim 10 \mathrm{kV}$, extend the non-destructive depth profile methodology.

\section{Acknowledgements}

Arie Knoester is thanked for help with the LEIS data collection and analysis. 


\section{References}

1. Moreau, P., et al. Influence of zeolite structure on ethylbenzene transformation. in 4th International FEZA Conference. 2008. Elsevier.

2. Mihindou-Koumba, P.C., et al., Methylcyclohexane transformation over H-EU-1 zeolite: Selectivity and catalytic role of the acid sites located at the pore mouths. Journal of Catalysis, 2008. 255(2): p. 324-334.

3. Magnoux, P., M. Guisnet, and I. Ferino, Pore mouth catalysis over acidic zeolites. Nature of active species, A. Corma, et al., Editors. 2000, Elsevier. p. 275-280.

4. Gruenert, W., et al., Investigations of Zeolites by Photoelectron and lon Scattering Spectroscopy 2. A New Interpretation of XPS Binding Energy Shifts in Zeolites. The Journal of Physical Chemistry, 1994. 98(42): p. 10920-10929.

5. Gijzeman, O.L.J., et al., The Effect of Chemical Composition and Structure on XPS Binding Energies in Zeolites. The Journal of Physical Chemistry B, 2003. 107(3): p. 678-684.

6. Barr, T.L., The nature of the relative bonding chemistry in zeolites: An XPS study. Zeolites, 1990. 10(8): p. 760-765

7. Barr, T.L. and M.A. Lishka, ESCA studies of the surface chemistry of zeolites. Journal of the American Chemical Society, 1986. 108(12): p. 3178-3186.

8. Grunert, W., M. Muhler, and H.G. Karge, Investigations of zeolites by photoelectron spectroacopy and ion-scattering spectroscopy. J. Chem. Soc. Soc., Faraday Trans., 1996. 92(4): p. 701-706.

9. Briggs, D. and M.P. Seah, Practical Surface Analysis by Auger and X-ray Photoelectron Spectroscopy1983, Chichester: Wiley.

10. Fadley, C.S., et al., Surface analysis and angular distributions in x-ray photoelectron spectroscopy. Journal of Electron Spectroscopy and Related Phenomena, 1974. 4(2): p. 93-137.

11. Baird, R.J., et al., Concentration profiles for irregular surfaces from $x$-ray photoelectron angular distributions. Analytical Chemistry, 1976. 48(6): p. 843-846. 
12. Gunter, P.L.J. and J.W. Niemantsverdriet, Thickness determination of uniform overlayers on rough substrates by angle-dependent XPS. Applied Surface Science, 1995. 89(1): p. 69-76.

13. Zier, M., et al., Non-destructive depth profile analysis using synchrotron radiation excited XPS. Microchim Acta, 2007. 156: p. 99-101.

14. Tao, F., et al., Reaction-Driven Restructuring of Rh-Pd and Pt-Pd Core-Shell Nanoparticles. Science, 2008. 322(5903): p. 932-934

15. Salmeron, M. and R. Schlögl, Ambient pressure photoelectron spectroscopy: A new tool for surface science and nanotechnology. Surface Science Reports, 2008. 63(4): p. 169-199.

16. Shimada, H., et al., Determination of external surface composition of zeolite particles by synchrotron radiation XPS. Catalysis Letters, 1996. 39: p. 125-128.

17. Shimada, H., et al., XPS depth profiling of powdered materials. Applied Surface Science, 1999. 144-145(0): p. 21-25.

18. Shimada, H., et al., XPS analysis of zeolitic materials. Journal of Surface Analysis, 1999. 5: p. 304307.

19. Degnan, T., Jr., Applications of zeolites in petroleum refining. Topics in Catalysis, 2000. 13(4): p. 349-356.

20. Kulprathipanja, S., ed. Zeolites in Industrial Separation and Catalysis. 2010, Wiley-VCH: Weinheim, Germany.

21. Knop-Gericke, A., et al., X-Ray Photoelectron Spectroscopy for Investigation of Heterogeneous Catalytic Processes. Adv. Catal., 2009. 52: p. 213-272.

22. Jablonski, A., F. Salvat, and C.J. Powell, Phys. CHem. Ref. Data, 2004. 33: p. 409-.

23. http://www.helmholtz-

berlin.de/pubbin/igama output?modus=einzel\&sprache=de\&gid=1607\&typoid=50740. 6/2/2015]

24. http://www.iontof.com. [cited 2015. 
25. Brongersma, H.H., et al., Surface composition analysis by low-energy ion scattering. Surface Science Reports, 2007. 62(3): p. 63-109.

26. Brongersma, H.H., Low-Energy lon Scatering, in Characterization of Materials, E.N. Kaufmann, Editor 2012, Wiley. p. 2024-2044.

27. Wang, D., J.H. Lunsford, and M.P. Rosynek, Characterization of a Mo/ZSM-5 Catalyst for the Conversion of Methane to Benzene. Journal of Catalysis, 1997. 169(1): p. 347-358.

28. Karge, H.G., B. Wichterlova, and H.K. Beyer, High-temperature interaction of solid Cu chlorides and $\mathrm{Cu}$ oxides in mixtures with $\mathrm{H}$-forms of ZSM-5 and Y zeolites. Journal of the Chemical Society, Faraday Transactions, 1992. 88(9): p. 1345-1351.

29. Grünert, W., R. Schlögl, and H.G. Karge, Photoelectron and ion scattering spectroscopy of zeolites under reduced surface-charge conditions. Surface and Interface Analysis, 1993. 20(7): p. 603-606.

30. Brongersma, H.H., P.A.C. Groenen, and J.-P. Jacobs, Application of low-energy ion scattering to oxidic surfaces, J. Nowotny, Editor 1994, Elsevier. p. 113-182.

31. Tanuma, S., C.J. Powell, and D.R. Penn, Calculations of electron inelastic mean free paths. III. Data for 15 inorganic compounds over the 50-2000 eV range. Surface and Interface Analysis, 1991. 17(13): p. 927-939.

32. Ceelen, W.C.A.N., et al., Caesium diffusion in sodium borosilicate glass studied by low-energy ion scattering. Surface and Interface Analysis, 1995. 23(10): p. 712-716. 